\title{
O CUSTO DE IMPERFEIÇÕES \\ DO MERCADO BRASILEIRO DE CAPITAIS: UMA APLICACÃO DA MODERNA TEORIA DE FINANÇAS
}

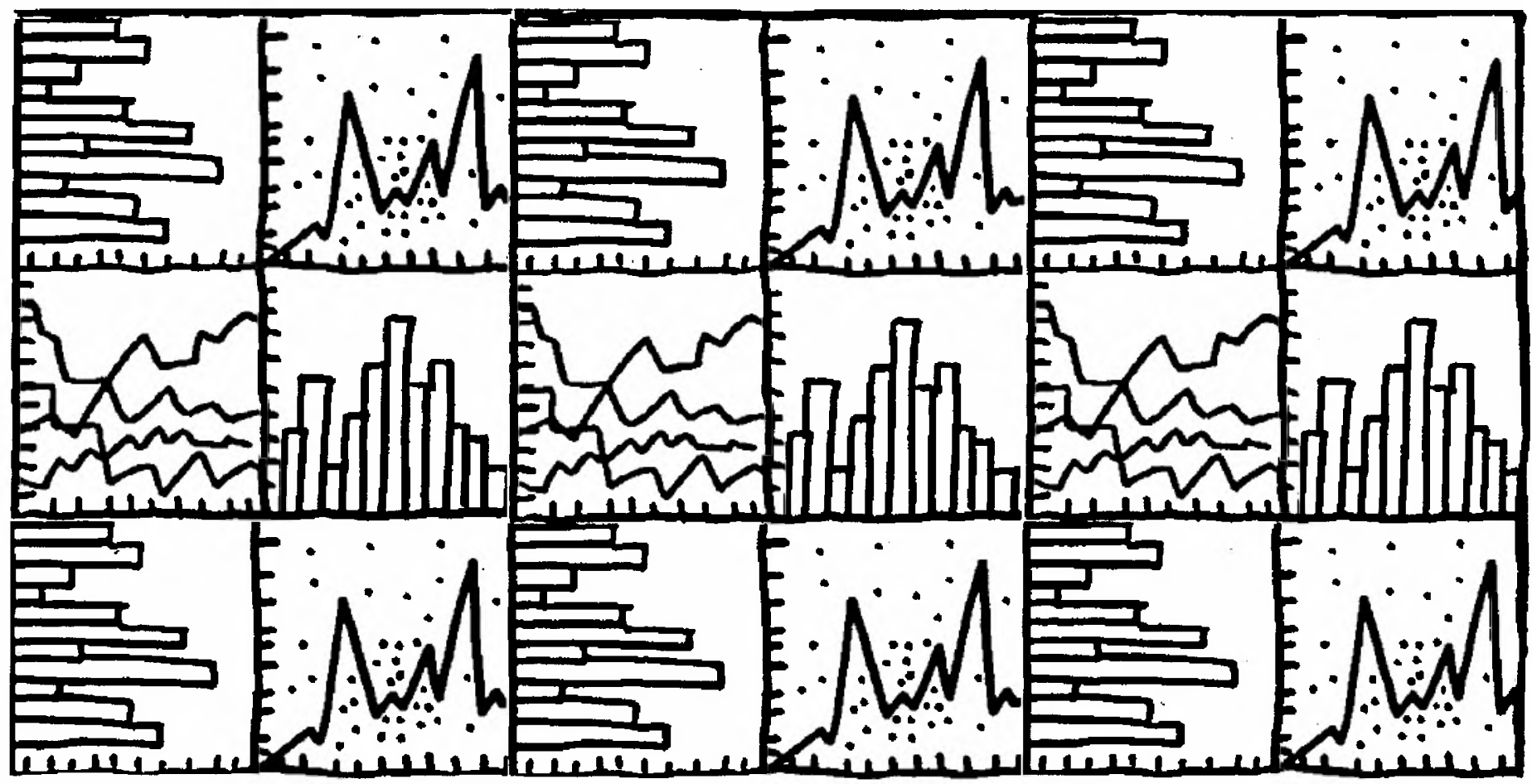

Antonio Zoratto Sanvicente

Professor Titular em Fînanças do Departamento de Administração da FEA-USP.

* RESUMO: Com dados de empresas com ações negociadas na Bolsa de Valores de São Paulo, relativos ao final de 1988, a relação entre três paradigmas fundamentais da moderna teoria de Finanças: - a análise ModiglianiMiller, o "capital asset pricing model" e o modelo de avaliação de opções (fórmula Black-Scholes) -, graças à obtenção de uma expressão para o custo médio de capital de uma empresa, é aplicada à avaliação (1) do custo de falência implicito nas estruturas de capital escolhidas pelas empresas, e (2) da significância da alegada falta de modalidades de financiamento a longo prazo. A conclusão final é a de que o custo de falência resulta em um acréscimo médio de custo de $1 \%$ ao mês, em termos reais, e que a falta de instrumentos de financiamento a longo prazo não implica em custos significativos.

* PALAVRAS-CHAVE: Estrutura de capital, risco sistemático e custo de capital, estimação de custos de falên- cia, prazos de financiamento de empresas, "option pricing model".

* ABSTRACT: Using information on companies with shares traded on the São Paulo Stock Exchange, relative to the end of the 1988 fiscal year, the relationship between the Modigliani-Miller capital structure analysis, the capital asset pricing model, and the Black-Scholes option pricing model, involving an equation for the firm's weighted cost of capital, is used for (1) estimating the cost of financial distress implied by the firm's choice of capital structure and (2) evaluating the impact of the absence of longer-term financing opportunities in the Brazilian capital market. The final results indicate that the cost of financial distress results in a one percent monthly addition to the cost of capital, in real terms, whereas the lack of longer-term financing has no significant economic consequences.

* KEY WORDS: Capital structure, systematic risk and cost of capital, estimation of costs of financial distress, long-term corporate financing, option pricing model. 


\section{INTRODUÇÃO}

A maior parte da "moderna teoria de Finanças" atualmente utilizada para o estabelecimento de princípios de administração financeira e análise de investimentos, descrita magistralmente em livros tais como os de Copeland e Weston ${ }^{1}$ e Brealey e Myers', baseia-se na "hipótese de mercado perfeito".

Isso quer dizer que quase todos os principais resultados que conhecemos dependem da suposição de que:

a) não há custos de transação ou impostos;

b) todos os participantes possuem as mesmas informações, que podem ser obtidas a custo zero.

Por exemplo, modelos consagrados como capital asset pricing model e option pricing model partem dessas condições.

Por outro lado, não se pode afirmar que várias tentativas brilhantes de desenvolvimento da teoria de Finanças em condições de mercado imperfeito não tenham sido feitas: dentre apenas alguns exemplos, podemos citar Miller $^{3}$, que se preocupa com a inclusão dos impostos na análise da estrutura ótima de capital, Litzenberger e Ramaswamy ${ }^{4}$, que fazem o mesmo em relação à política de dividendos, e Brealey e Myers ${ }^{5}$, que analisam várias imperfeições de mercado (subsídios, custos de transação e impostos) que levam à falta de separação entre decisões de investimento e financiamento.

Neste trabalho, são descritas e relacionadas as principais análises da decisão de financiamento existentes na teoria de Finanças apoiada na hipótese de mercado perfeito, a saber, Modigliani e Miller ${ }^{6}$ capital asset pricing model e option pricing model.

Como estão baseadas na hipótese de mercado perfeito (excluindo-se a possibilidade de imposto sobre a renda das empresas, conforme Modigliani e Miller ${ }^{7}$, elas fazem predições específicas a respeito de qual deve ser o grau de endividamento ótimo de qualquer empresa, bem como o custo médio de capital resultante.

Entretanto, como todos sabemos, o mercado não é perfeito, e as empresas não mantêm o grau ótimo de endividamento previsto por essa teoria. Assim, este trabalho procura quantificar a diferença, para uma amostra de empresas que operam no Brasil, entre o endividamento determinado pela teoria e o endividamento observado.

Essa diferença é então atribuída a imperfeições de mercado, ou, mais especificamente, à existência de "custos de falência" e à inexistência de instrumentos de captação de recursos de terceiros de acordo nos prazos imaginados pelos modelos.

Portanto, medimos o quanto custam, para algumas empresas brasileiras, (a) a possibilidade de falência e (b) a falta relativa de financiamento a longo prazo. Até certo ponto, trata-se de uma aferição da robustez da teoria baseada na hipótese de mercado perfeito num mercado reconhecidamente imperfeito.

Para alcançar as suas finalidades, o trabalho está assim organizado: na sua segunda parte, é apresentada a teoria sobre a estrutura ótima de capital ${ }^{8}$, (capital asset pricing model e option pricing model), e esses vários paradigmas são combinados para gerar predições sobre o grau ótimo de endividamento de empresas.

$\mathrm{Na}$ terceira parte do trabalho, mede-se o custo médio de capital das empresas para aferir a significância de (a) custos de falência e (b) inexistência de alternativas de captação de recursos de terceiros aos prazos (longos) imaginados pela teoria. As diferenças então encontradas entre endividamento teórico e endividamento medido empiricamente, em suas implicações para o custo médio de capital da empresa, são quantificadas e analisadas estatisticamente.

Na quarta parte, o trabalho é concluído com uma proposta de extensão da análise a empresas com características distintas daquelas incluídas na amostra estudada.

\section{ALCUNS PARADIGMUS DA MODERMA TEORIA DE FINANÇAS SOBRE A ESTRUTURA OTIMA DE CAPTTAL DA EMPRESA}

\section{Definição dos símbolos a serem utiliza-} dos:

$\mathbf{k}_{\mathbf{m}}=$ custo médio de capital da empresa, depois do IRPJ; IRPI;

$\mathbf{K}_{\mathbf{d}}=$ custo do capital de terceiros, antes do

$\mathbf{k}_{\mathbf{s}}=$ custo do capital próprio, antes (e depois) do IRPJ;

$\mathbf{F}=$ valor de mercado do capital de terceiros usado pela empresa;

$S$ = valor de mercado do capital próprio usado pela empresa;

$\mathbf{V}=\mathrm{F}+\mathrm{S}=$ valor total de mercado da empresa (isto é, ativo total);

$\mathbf{L}=\mathrm{F} / \mathrm{V}=$ endividamento geral da empresa;

$\mathbf{R}_{\mathbf{F}}=$ taxa de retorno de ativo livre de risco;

$\beta_{\mathrm{V}}=$ coeficiente de risco sistemático (beta) dos ativos da empresa;

$\beta_{S}=$ coeficiente de risco sistemático do 
capital próprio da empresa;

$\beta_{\mathbf{D}}=$ coeficiente de risco sistemático do capital de terceiros usado pela empresa;

$\mathbf{E}\left(\mathbf{R}_{\mathbf{m}}\right)=$ taxa esperada de retorno da "carteira teórica de mercado", ou seja, a carteira m;

$\mathbf{t}=$ alíquota do Imposto sobre a Renda de Pessoas Jurídicas;

$\mathbf{N}\left(\mathrm{d}_{1}\right)=$ probabilidade acumulada, na distribuição normal reduzida, de $-\infty \mathrm{a} \mathrm{d}_{1}$,

onde $d_{1}=\left\{\left[\ln (V / B)+R_{F} T\right] / \sigma_{V} T^{0,5}\right\}+$ $+\sigma_{\mathrm{V}} \mathrm{T}^{0,5}$, sendo:

$\mathbf{B}=$ valor de resgate das dívidas da empresa;

$\mathbf{T}=$ prazo remanescente até a data de vencimento das dívidas da empresa;

$\sigma_{\mathbf{V}}=$ desvio-padrão da taxa de retorno sobre o ativo total da empresa.

\section{Custo médio de capital da empresa}

Segundo Modigliani e Miller', e utilizando os símbolos acima,

$$
k_{m}=k_{d}(1-t) L+k_{s}(1-L)
$$

$\mathrm{Na}$ análise desses autores, feita anteriormente em 1958, $t=0$, por hipótese, concluía-se que o valor de mercado da empresa (V), independia de $L$, ou seja, não haveria estrutura ótima de capital. Note-se que maximizar $V$ é a mesma coisa que minimizar $\mathrm{k}_{\mathrm{m}}$.

As hipóteses feitas por Modigliani e Miller para chegar a essa conclusão foram:

a) o mercado de capitais é perfeito;

b) os indivíduos podem emprestar e tomar emprestado à taxa de retorno do ativo livre de risco;

c) não há custos de falência;

d) as empresas emitem apenas dois tipos de títulos: debêntures livres de risco e ações (que possuem risco);

e) todas as empresas pertencem à mesma classe de risco;

f) todos os fluxos de caixa são perpétuos e uniformes (isto é, não há crescimento).

3. Custo médio de capital, capital asset pricing model e option pricing model

Segundo o capital asset pricing model desenvolvido por Sharpe ${ }^{10,}$ a taxa de retorno exigida, em equilíbrio, por um investidor em ações e, portanto, o custo de capital próprio da empresa à qual esse investidor fornece seu capital, é dada por:

$$
k_{s}=R_{F}+\left[E\left(R_{m}\right)-R_{F}\right] \beta^{L}
$$

onde, além dos símbolos já definidos anteriormente, temos $\beta \mathrm{L}$, que indica o coeficiente de risco sistemático de ações de uma empresa que tem capital de terceiros em sua estrutura de capital.

Por sua vez, a relação entre esse coeficiente e o risco sistemático dos ativos da empresa é dada pelo option pricing model ${ }^{11}$.

$$
\beta^{L}=N\left(d_{1}\right)(V / S) \beta V
$$

Portanto, podemos escrever o custo de capital próprio substituindo (3) em (2), obtendo:

$$
k_{s}=R_{F}+\left[E\left(R_{m}\right)-R_{F}\right] N\left(d_{1}\right)[1 /(1-L)] \beta_{V}
$$

notando-se que $\mathrm{V} / \mathrm{S}=1 /(1-\mathrm{L})$.

Por meio de análise semelhante, chega-se à seguinte expressão para o custo do capital de terceiros, antes do IRPJ:

$$
k_{d}=R_{F}+\left[E\left(R_{m}\right)-R_{F}\right] N\left(-d_{1}\right)(1 / L) \beta V
$$

Finalmente, substituindo $k_{s}$ e $k_{d}$ em (1) por suas expressões dadas por (4) e (5), usando (3), e simplificando, obtém-se:

$k_{m}=R_{F}(1-t L)+\left[E\left(R_{m}\right)-R_{F}\right] L^{L} / N\left(d_{1}\right)\left[1-t+t N\left(d_{1}\right)\right](1-L)$

\section{Discussão da equação (6)}

A equação (6) permite-nos discutir alguns dos principais resultados da teoria da decisão de financiamento encontrados na literatura de Finanças.

I) Se não houver imposto de renda $(t=0) \mathrm{e}$ capital de terceiros não tiver risco $\left(N\left(d_{1}\right)=1\right)$ :

Nesse caso, a equação (6) reduz-se a:
9. Idem, ibidem.

10. SHARPE, W.F. "Capital Asset Prices: A Theory of Market Equilibrium under Conditions of Risk". Journal of Finance, setembro de 1964, pp.425-42.

11. Ver GALAI, D. \& MASULIS, R. "The Option Pricing Model and the Risk Factor of Stock". Journal of Financial Economics, janeiromarço de 1976, pp.53-82. 


$$
k_{m}=R_{F}+\left[E\left(R_{m}\right)-R_{F}\right] \beta_{V}
$$

O resultado obtido na equação (7) é exatamente o apresentado por Modigliani e Miller $^{12}$. Note-se que $\left(\beta^{L}\right)(1-L)$ foi substituído por $\beta_{\mathbf{V}}$, o que é permitido pela equação (3), quando $\mathrm{N}\left(\mathrm{d}_{1}\right)$ é igual a 1 . Como indica a equação (7), e argumentavam Modigliani e Miller em seu primeiro artigo, o valor da empresa (inversamente relacionado ao custo médio de capital) independe da estrutura de capital, já que L não aparece na equação. E o custo médio é o mesmo para qualquer grau de endividamento, sendo decorrente da "classe de risco" à qual pertence a empresa, que nesse caso é definida pelo valor do coeficiente de risco sistemático de seus ativos, $\beta_{\mathrm{V}}$. $t>0$ :

II) Se capital de terceiros não tem risco e

Nesse caso, a equação (6) é substituída por:

12.MODIGLIANI, F. \& MILLER, M. "The Cost of Capital, Corporation Finance and the Theory of Investment". Op. cit.

13. MODIGLIANI, F. \& MILLER, M. "Taxes and the Cost of Capital: A Correction". Op. cit.

14. Para uma explicacão ver, por exemplo, SANVICENTE, A.Z. Corporate Bond Prices in an Option Pricing Framework. Stanford, Ca., tese de doutoramento, Stanford University, 1982.

15. HSIA, C. "Relationships among the Theories of MM, CAPM, and OPM". (Manuscrito.) UCLA, Los Angeles, 1978; apud COPELAND T.E. \& WESTON, J.F. Op. cit., cap. 8.

16. MODIGLIANI, F. \& MILLER, M. "Taxes and the Cost of Capital: A Correction". Op. cit.

17. BRADLEY, M.; JARRELL, G., \& KIM, E.H. "On the Existence of an Optimal Capital Structure: Theory and Evidence". Journal of Finance, julho de 1984 pp.857-78.

Vemos, portanto, que o custo médio de capital da empresa deixa de ser independente de L (grau de endividamento), e que, quanto maior o valor de $\mathrm{L}$, menor será o custo médio de capital. Portanto, a estrutura ótima de capital será aquela na qual $L=1$ (ou seja, 100\% de capital de terceiros), tal como obtido por Modigliani e Miller ${ }^{13}$. Isso é devido à vantagem fiscal existente com a utilização de capital de terceiros (por causa da dedutibilidade das despesas financeiras), não compensada por aumentos de risco, já que, por hipótese, $o$ capital de terceiros não tem risco.

\section{Imperfeições de mercado}

Para discutir a influência de imperfeições de mercado sobre a relação entre valor da empresa e estrutura de capital, um dos paradigmas mais apropriados é o chamado option pricing model ${ }^{14}$.
Segundo foi demonstrado também por Hsia' ${ }^{15}$, admitindo a hipótese de que o capital de terceiros tivesse risco, ou seja, que houvesse "risco de falência", com o que a nossa equação básica, isto é, a equação (6), incluiria um valor $\mathrm{N}\left(\mathrm{d}_{1}\right)<1$, chegar-se-ia ao mesmo resultado de Modigliani e Miller ${ }^{16}$. Em outras palavras, Hsia mostrou que o resultado básico não exigia a hipótese de capital de terceiros sem risco.

Nossa análise da equação (6) indica, porém, que $\left[1-t+t N\left(d_{1}\right)\right]$ precisa ser positivo para que o custo médio de capital seja minimizado com $\mathrm{L}=1$. Mas, desde que $t<1$, que corresponde à realidade, essa condição sempre se verifica.

Por outro lado, a análise de Modigliani e Miller deixa de apresentar os resultados anteriores quando há, além de "risco de falência", o que iremos chamar de "custo de falência", para traduzir a expressão costs of financial distress, conforme Bradley, Jarrell e Kim ${ }^{17}$.

Segundo esses autores, essa expressão abrange:

a) custos da relação de agency entre acionistas e credores, decorrentes da renegociação de contratos de financiamento da empresa, e os custos da tomada de decisões subótimas de produção e investimento, que resultam da situação de financial distress, que é tanto mais grave quanto mais elevado é o grau de endividamento da empresa;

b) custos diretos $e$ indiretos de falência (por exemplo, despesas com procedimentos judiciais e legais, no primeiro caso, e perdas pela liquidação de ativos a preços inferiores aos valores que alcançariam se a empresa continuasse em funcionamento normal).
6. Predições da teoria de mercado perfeito

Para prosseguir com a análise empírica das políticas de endividamento das empresas no mercado brasileiro de capitais, é útil definir alguns valores de referência do custo médio de capital, o que é feito a seguir.

\subsection{Se $\mathrm{L}=1$ e $\mathrm{T}$ tender $\mathrm{a}+\infty$.}

Já foi verificado que, se houver IRPJ $(t>0)$, e as despesas financeiras forem dedutíveis, a estrutura ótima de capital, com ou sem risco para as dívidas (mas sem custo de falência), será representada pela escolha de $L=1$ pela empresa.

Se, além disso, $\mathrm{T}$ tender a infinito (o que é equivalente à suposição de Modigliani e Miller, de que o capital de terceiros não tem risco), obtemos $\mathrm{N}\left(\mathrm{d}_{1}\right)=1$, por um bem conhecido resultado do option pricing model. 
Nesse caso, o custo médio de capital é igual à taxa de retorno do ativo sem risco, ajustada pela alíquota do IRPJ, como se pode ver na equação (8). Ou seja,

$$
k_{m 1}=R_{F}(1-t)
$$

6.2. Se $\mathrm{T}$ tender $\mathrm{a}+\infty$, mas $\mathrm{L}<1$.

Pode ocorrer, porém, que por limitações institucionais ou econômicas do mercado de capitais, que podem muito bem ser respostas racionais a custos de falência, as empresas não consigam alcançar o grau de endividamento que a teoria diz que seria ótimo, ou seja, $L=1$, e que efetivamente $L<1$.

Pelas condições deste item, o custo médio de capital também é dado pela equação (8), e passamos a chamar essa taxa de $k_{m 2}$, a qual, como pode ser percebido na equação, é superior a $\mathrm{k}_{\mathrm{m} 1}$, já que todos os termos da segunda parcela de (8) são positivos, nesse caso.

Essa taxa será utilizada para se aferir a magnitude dos custos de falência implícitos nos dados relativos às estruturas de capitais de empresas brasileiras.

\subsection{Se $\mathrm{T}$ for finito $\mathrm{e} \mathrm{L}<1$}

Este é o caso que mais se aproxima da situação real, sendo representado pelo custo médio de capital dado pela equação (6); agora, $N\left(d_{1}\right)<1$. Essa taxa será simbolizada por $k_{m 3}$, sendo possível verificar que essa taxa é superior a $k_{\mathrm{m} 2}$.

\section{RESULTADOS DA ANÁLISE EMPÍRICA}

\section{Dados utilizados}

Para que se pudessem obter resultados numéricos com o uso das equações (6) e (8), as diversas variáveis foram medidas da seguinte maneira:

$\mathbf{R}_{\mathbf{F}}=$ taxa de juros mensal prometida em saldos de caderneta de poupança, ou seja, 0,005 .

$\left[\mathbf{E}\left(\mathbf{R}_{\mathrm{m}}\right)-\mathbf{R}_{\mathrm{F}}\right]=$ prêmio de mercado por unidade de risco sistemático, estimado em Sanvicente $^{18}$, e igual a 0,013 , ou seja, $1,3 \%$ ao mês, em função das condições vigentes no mercado de ações da Bolsa de Valores de São Paulo em 31 de julho de 1988.

$\beta^{L}=$ estimado através de regressões entre a lucratividade mensal de cada ação e a lucratividade mensal do Índice Bovespa no período de janeiro de 1986 a dezembro de 1988.

$\mathbf{N}\left(\mathbf{d}_{1}\right)=$ estimado com o uso das seguintes informações:

$\mathbf{V} / \mathbf{B}=1 /$ índice de endividamento geral (contábil) de cada empresa, segundo balanços patrimoniais relativos ao exercício de 1988; onde $\mathbf{B}=$ saldo contábil de todas as exigibilidades de cada empresa;

$\mathbf{R}_{\mathrm{F}}=0,005$

$T=30,60,90$ ou 120 meses, conforme o necessário para fins de análise;

$\sigma_{\mathrm{V}}=$ em função do desvio-padrão da taxa de retorno da ação de cada empresa $\left(\sigma_{S}\right)$, usando lucratividades mensais no período de janeiro de 1986 a dezembro de 1988, e a partir da seguinte relação:

$$
\sigma_{V}^{2}=\sigma_{S}^{2} /[1+(1-t)(B / P)]^{2}
$$

onde:

$\mathbf{P}=$ patrimônio líquido total da empresa.

$\mathbf{t}=0,35$ (alíquota marginal do IRPJ).

\section{Resultados obtidos}

A tabela 1 contém os valores mensais estimados para as taxas $\mathrm{k}_{\mathrm{m} 2}$ e $\mathrm{k}_{\mathrm{m} 3}$, esta última para $\mathrm{T}=30$ e 120 meses, por empresa. Como se observa, a amostra contém 54 empresas.

\section{Testes de hipóteses}

3.1. Há custo de falência e é significativo ?

$\mathrm{O}$ primeiro teste refere-se à verificação da existência de custos de falência significativos. Para esse fim, foram comparadas as taxas $\mathrm{k}_{\mathrm{m} 2}$ (média para as empresas da amostra), e $k_{m 1}=R_{F}(1-t)=0,005(1-0,35)=0,00325$.

$\mathrm{O}$ valor médio obtido para a taxa $\mathrm{k}_{\mathrm{m} 2}$ foi de 0,013584 (ou 1,3584\% ao mês), e o desviopadrão estimado é de $0,002889(0,2889 \%$ ao mês). Portanto, o valor calculado da estatística $t$, supondo que as taxas tenham distribuição normal, e para a hipótese nula de custo médio de capital, depois do IRPJ, igual a $0,325 \%$ ao mês, é igual a 3,577 . Ou seja, a probabilidade de que o valor médio de $\mathbf{k}_{\mathrm{m} 2}$, nas empresas da amostra, se iguale a 0,00325, não chega a 0,001 (uma chance em mil). Portanto, há custos de falência, e eles alcançavam
18. SANVICENTE, A.Z. "Avaliação de Empresas". São Paulo, FEA-USP, 1990 (manuscrito). 
Tabela 1: Estimativas de custo médio de capital, em função de algumas suposições sobre o prazo médio de vencimento das dívidas da empresa

\begin{tabular}{|c|c|c|c|}
\hline Empresa & $\begin{array}{c}\mathrm{T}= \\
\text { intinilo }\end{array}$ & $\begin{array}{c}\mathrm{T}= \\
30 \text { meses }\end{array}$ & $\begin{array}{c}T= \\
120 \text { meses }\end{array}$ \\
\hline Aços Villares & 1,0041\% a.m. & $0,6225 \%$ a.m. & $0,6173 \%$ a.m. \\
\hline Belgo Mineira & 1,6090 & 0,8256 & 0,8239 \\
\hline Brahma & 1,1833 & 0,6889 & 0,6859 \\
\hline Brasmotor & 1,2709 & 0,7249 & 0,7256 \\
\hline Cacique & 1,2243 & 0,7064 & 0,7048 \\
\hline Caemi & 1,5244 & 0,7989 & 0,7990 \\
\hline CBV Inds. Mecs. & 1,1621 & 0,6858 & 0,6755 \\
\hline Ceval & 0,9204 & 0,6005 & 0,5868 \\
\hline Cimento Itaú & 1,4632 & 0,7817 & 0,7801 \\
\hline Cobrasma & 1,1833 & 0,6955 & 0,6801 \\
\hline Cofap & 1,3432 & 0,7530 & 0,7347 \\
\hline Confab & 1,3179 & 0,7397 & 0,7274 \\
\hline Copene & 1,8960 & 0,9200 & 0,9136 \\
\hline Cosigua & 1,3430 & 0,7519 & 0,7348 \\
\hline Cruzeiro do Sul & 0,8453 & 0,5766 & 0,5724 \\
\hline Duratex & 1,5788 & 0,8210 & 0,8135 \\
\hline Eluma & 1,6567 & 0,8477 & 0,8378 \\
\hline Estrela & 1,4229 & 0,7693 & 0,7639 \\
\hline Eucalex & 1,5511 & 0,8134 & 0,8031 \\
\hline Ferbasa & 1,7759 & 0,8775 & 0,8762 \\
\hline Ferro Brasileiro & 1,3256 & 0,7415 & 0,7382 \\
\hline Ferro Ligas & 1,4975 & 0,7963 & 0,7870 \\
\hline FNV & 1,9644 & 0,9281 & 0,9086 \\
\hline Frigobrás & 1,0853 & 0,6601 & 0,6472 \\
\hline Klabin & 1,3380 & 0,7372 & 0,7359 \\
\hline Mannesmann & 1,5467 & 0,8180 & 0,8025 \\
\hline Marcopolo & 1,5356 & 0,8141 & 0,7926 \\
\hline Massey Perkins & 1,1247 & 0,6719 & 0,6550 \\
\hline Mendes Jr. & 0,8676 & 0,5855 & 0,5651 \\
\hline Mesbla & 0,8681 & 0,5955 & 0,5955 \\
\hline Metal Leve & 1,3030 & 0,7232 & 0,7359 \\
\hline Moinho Santista & 1,2342 & 0,6995 & 0,7008 \\
\hline Olvebra & 2,1560 & 0,9975 & 0,9944 \\
\hline Paraibuna & 1,2685 & 0,7226 & 0,7109 \\
\hline Paranapanema & 1,6124 & 0,8264 & 0,8244 \\
\hline Perdigäo Alims. & 1,5678 & 0,8172 & 0,8135 \\
\hline Petrobrás & 1,1963 & 0,6930 & 0,6857 \\
\hline Petróleo lpiranga & 1,4959 & 0,8012 & 0,7831 \\
\hline Pirelli & 1,4416 & 0,7835 & 0,7665 \\
\hline Polipropileno & 1,5520 & 0,8131 & 0,8041 \\
\hline Prometal & 1,8021 & 0,8964 & 0,8811 \\
\hline Refripar & 1,0048 & 0,6306 & 0,6199 \\
\hline Sadia Concórdia & 1,6275 & 0,8379 & 0,8213 \\
\hline Samitri & 1,5413 & 0,8021 & 0,8025 \\
\hline Sharp & 1,1245 & 0,6754 & 0,6602 \\
\hline Sid Informálica & 1,0354 & 0,6410 & 0,6314 \\
\hline Sid. Riograndense & 1,4774 & 0,7875 & 0,7815 \\
\hline Souza Cruz & 1,2406 & 0,7117 & 0,7129 \\
\hline Suzano & 1,5252 & 0,7972 & 0,7969 \\
\hline Tupy & 1,3530 & 0,7492 & 0,7425 \\
\hline Vale Rio Doce & 1,0387 & 0,6411 & 0,6299 \\
\hline Varig & 0,6197 & 0,4661 & 0,4625 \\
\hline Vidr. Sta. Marina & 1,2763 & 0,7146 & 0,7143 \\
\hline White Martins & 1,4292 & 0,7712 & 0,7650 \\
\hline Média & 1,3584 & 0,7472 & 0,7391 \\
\hline Desvio-padrăo & 0,2889 & 0,0992 & 0,0993 \\
\hline
\end{tabular}

aproximadamente $1 \%$ ao mês, em termos reais, em fins de 1988.

3.2. Quanto custa a falta de financiamento a longo prazo ?

$\mathrm{O}$ segundo teste procura verificar se a tão criticada falta de mecanismos de financiamento (com recursos de terceiros) a longo prazo é significativa, em termos de custo de capital. Para esse fim, foi feita uma comparação entre as médias obtidas para $\mathrm{k}_{\mathrm{m} 3}$, $\operatorname{com} \mathrm{T}=$ 30 e 120 meses.

Fazendo-se uso do instrumental desenvolvido neste trabalho, a impossibilidade de contar com um perfil de endividamento mais alongado deveria ter se manifestado em custo médio de capital mais elevado. Objetivamente, o custo médio com $\mathrm{T}=30$ meses deveria ter sido maior do que o custo médio $\operatorname{com} \mathrm{T}=120$ meses.

$\mathrm{E}$ isso de fato ocorre, com as empresas contidas na amostra $(0,7472 \%$ vs. $0,7391 \%$ ao mês, para a média da amostra), conforme aponta a tabela. Entretanto, essa diferença não é significativa, já que produz uma estatística t calculada de apenas 0,4241 .

Portanto, conclui-se que esse tão comentado problema de nosso mercado de capitais talvez não seja realmente significativo.

\section{CONCLUSÕES}

Neste trabalho, que estimou os custos médios de capital de empresas com ações negociadas em bolsa de valores, foi constatado que há custos de falência significativos, e seu efeito em termos de custo de capital foi estimado. Também se verificou que a falta de instrumentos de captação de recursos de terceiros com prazos mais longos não é um problema sério a ponto de ter efeitos significativos sobre o custo médio de capital das empresas.

E de suspeitar-se, porém, que a amostra escolhida tenha levado ao segundo resultado obtido. Por força de sua posição como empresas abertas e com ações negociadas em bolsa de valores, as empresas da amostra já contam com (a) maior facilidade de acesso a capital próprio (de prazo indeterminado, ou seja, infinitamente longo), e (b) com a possibilidade de emitir debêntures.

Para empresas semelhantes, porém fechadas, dalvez haja um impacto significativo. Em pesquisa posterior, a mesma metodologia será aplicada com vistas à complementação dessas constataçōes sobre características reais do mercado brasileiro de capitais. $\square$ 